Nota científica

\title{
Primer reporte de Eotetranychus lewisi en la región andina del Ecuador en Arracacia xanthorrhiza (zanahoria blanca) y Tropaeolum tuberosum (mashua)
}

\author{
First report of Eotetranychus lewisi in the Andean region from Ecuador on Arracacia xanthorrhiza \\ (white carrot), Tropaeolum tuberosum (mashua) \\ Carlos Vásquez ${ }^{\mathrm{a}, *}$, Marta Dávila ${ }^{\mathrm{a}}$, Norma Telenchana ${ }^{\mathrm{a}}$, José Mangui ${ }^{\mathrm{a}}$ y Daniela Navas ${ }^{\mathrm{b}}$ \\ ${ }^{a}$ Facultad de Ciencias Agropecuarias, Universidad Técnica de Ambato, Carretera Cevallos-Quero, código postal 180350, Cevallos, Tungurahua, Ecuador \\ ${ }^{\mathrm{b}}$ Facultad de Ciencia e Ingeniería en Alimentos, Universidad Técnica de Ambato, Av. Los Atis, Campus Huachi, código postal 180150, Ambato, Tungurahua, \\ Ecuador
}

Recibido el 23 de enero de 2017; aceptado el 26 de mayo de 2017

Disponible en Internet el 6 de diciembre de 2017

\section{Resumen}

Eotetranychus lewisi (McGregor) es registrado por primera vez sobre plantas de zanahoria blanca (Arracacia xanthorrhiza Bancr.) y mashua (Tropaeolum tuberosum Ruiz y Pavon) en la Provincia de Tungurahua, Ecuador. Los mayores niveles poblacionales de E. lewisi fueron observados sobre tipos de A. xanthorrhiza de pecíolos y nervaduras color púrpura, mientras que sobre mashua solo fue observado un pequeño número de estos ácaros. Las observaciones se realizaron en material sembrado en la Facultad de Ciencias Agropecuarias de la Universidad Técnica de Ambato. Basado en la importancia económica de la zanahoria blanca en las comunidades rurales ecuatorianas se recomienda realizar estudios más detallados sobre el impacto potencial de E. lewisi en la sierra ecuatoriana.

(C) 2017 Universidad Nacional Autónoma de México, Instituto de Biología. Este es un artículo Open Access bajo la licencia CC BY-NC-ND (http://creativecommons.org/licenses/by-nc-nd/4.0/).

Palabras clave: Cultivos andinos; Tetranychidae; Fitófago

\section{Abstract}

Eotetranychus lewisi (McGregor) is reported for the first time on white carrot (Arracacia xanthorrhiza Bancr.) and mashua (Tropaeolum tuberosum Ruiz y Pavon) in Province of Tungurahua, Ecuador. Highest population levels of E. lewisi were observed on a purple petiolated and vein A. xanthorrhiza while on mashua only a lower number of this mite was registered. Observations were made at Facultad de Ciencias Agropecuarias, Universidad Técnica de Ambato. Based on economic importance of white carrot in rural communities it is suggested to carry out more detailed studies dealing potential impact of E. lewisi in the Ecuadorian Sierra.

(C) 2017 Universidad Nacional Autónoma de México, Instituto de Biología. This is an open access article under the CC BY-NC-ND license (http://creativecommons.org/licenses/by-nc-nd/4.0/).

Keywords: Andean crops; Tetranychidae; Phytophagous

\footnotetext{
* Autor para correspondencia.

Correo electrónico: ca.vasquez@uta.edu.ec (C. Vásquez).

La revisión por pares es responsabilidad de la Universidad Nacional Autónoma de México.
} 
Eotetranychus lewisi ha sido reportado en 71 especies de plantas hospederas dentro de 26 familias (Bolland, Gutiérrez y Flechtmann, 1998; Migeon, Nouguier y Dorkeld, 2011). De acuerdo con Vacante (2016), E. lewisi ocurre principalmente en la región neártica, donde se alimenta tanto de especies de plantas silvestres como cultivadas. Sin embargo, también se ha registrado en la región neotropical, incluyendo Bolivia, Chile, Colombia, Costa Rica, El Salvador, Guatemala, Honduras, Panamá y Perú (Bolland et al., 1998; Migeon et al., 2011). E. lewisi es considerada una plaga de importancia en poinsetias (Euphorbia pulcherrima Willd. ex Klotzsch), pero también ha sido observada causando daño en durazno (Prunus persica L.) en el norte de México (Pérez-Santiago et al., 2007), así como en cítricos, fresas y frambuesas (Howell y Daugovish, 2013; Jeppson, Keifer y Baker, 1975). Durante los últimos años se ha observado un incremento de las poblaciones de E. lewisi en cultivos de fresa, en California, probablemente debido a que el control químico y biológico aplicado para controlar Tetranychus urticae Koch haya liberado a E. lewisi de la competencia interespecífica (Howell y Daugovish, 2013).

En octubre del 2016 fueron realizados muestreos en el huerto experimental de la Facultad de Ciencias Agropecuarias de la Universidad Técnica de Ambato, Provincia de Tungurahua, en Ecuador (altitud de 2,850 m snm, y coordenadas geográficas $01^{\circ} 24^{\prime} 02^{\prime \prime} \mathrm{S}, 78^{\circ} 35^{\prime} 20^{\prime \prime} \mathrm{O}$ ), para detectar la presencia de ácaros fitófagos en algunas especies de cultivos andinos: amaranto (Amarathus quitensis: Amaranthaceae), mashua (Tropaeolum tuberosum: Tropaeolacae), miso o mauka (Mirabilis expansa: Nictaginaceae), papa andina (Solanum tuberosum var. andigena: Solanaceae), pepino dulce (Solanum muricatum: Solanacaeae) y sobre 2 accesiones de zanahoria blanca (Arracacia xanthorrhiza: Apiaceae).
La zona en estudio se caracteriza por una humedad relativa del $75.8 \%$, una precipitación anual de $494 \mathrm{~mm}$, una temperatura media diaria de $13.5^{\circ} \mathrm{C}$ y una velocidad del viento de $1.6 \mathrm{~m} / \mathrm{s}$ (http://www.serviciometeorologico.gob.ec/, consultado el $28 \mathrm{de}$ septiembre de 2016). Durante el muestreo fueron observadas poblaciones de ácaros tetraníquidos que fueron evidenciadas por los síntomas de amarillamiento en plantas de zanahoria blanca (fig. 1), mientras que en plantas de mashua las poblaciones eran menores, por lo que aún no se evidenciaban síntomas de alimentación. Las muestras de hojas infestadas con ácaros fueron llevadas al laboratorio de Sanidad Vegetal de la Facultad de Ciencias Agropecuarias y examinadas bajo aumento del microscopio estereoscópico, donde fueron observados todos los estados de desarrollo del ácaro. Posteriormente, los especímenes fueron montados usando líquido de Hoyer para observación al microscopio óptico.

La especie fue determinada como Eotetranychus lewisi (McGregor), basándose en la presencia de 2 pares de sedas anales, 2 pares de sedas paraanales y la morfología del edeago (fig. 2). La especie fue confirmada por el Dr. Carlos Flechtmann, de la Escola Superior de Agricultura Luiz de Queiroz, de la Universidade de São Paulo, en Brasil. Además de E. lewisi, fueron también observados ejemplares de T. urticae sobre la misma hoja. Adicionalmente, los mayores niveles poblacionales de E. lewisi fueron observados en plantas de A. xanthorrhiza de pecíolos y nervaduras color púrpura, provenientes de la parroquia Quinchicoto (municipio Tisaleo, Tungurahua) $\left(01^{\circ} 23^{\prime} 54^{\prime \prime}\right.$ $\mathrm{S}, 78^{\circ} 24^{\prime} 41^{\prime \prime} \mathrm{O}$; altura $3,260 \mathrm{~m} \mathrm{snm}$ ), mientras que $T$. urticae fue la especie predominante en el tipo de nervaduras y pecíolos verdes provenientes de la parroquia El Triunfo (municipio Baños, Tungurahua) $\left(01^{\circ} 10^{\prime} 18^{\prime \prime} \mathrm{S}, 78^{\circ} 32^{\prime} 33^{\prime \prime} \mathrm{O}\right.$, altura $1,683 \mathrm{~m} \mathrm{snm})$.

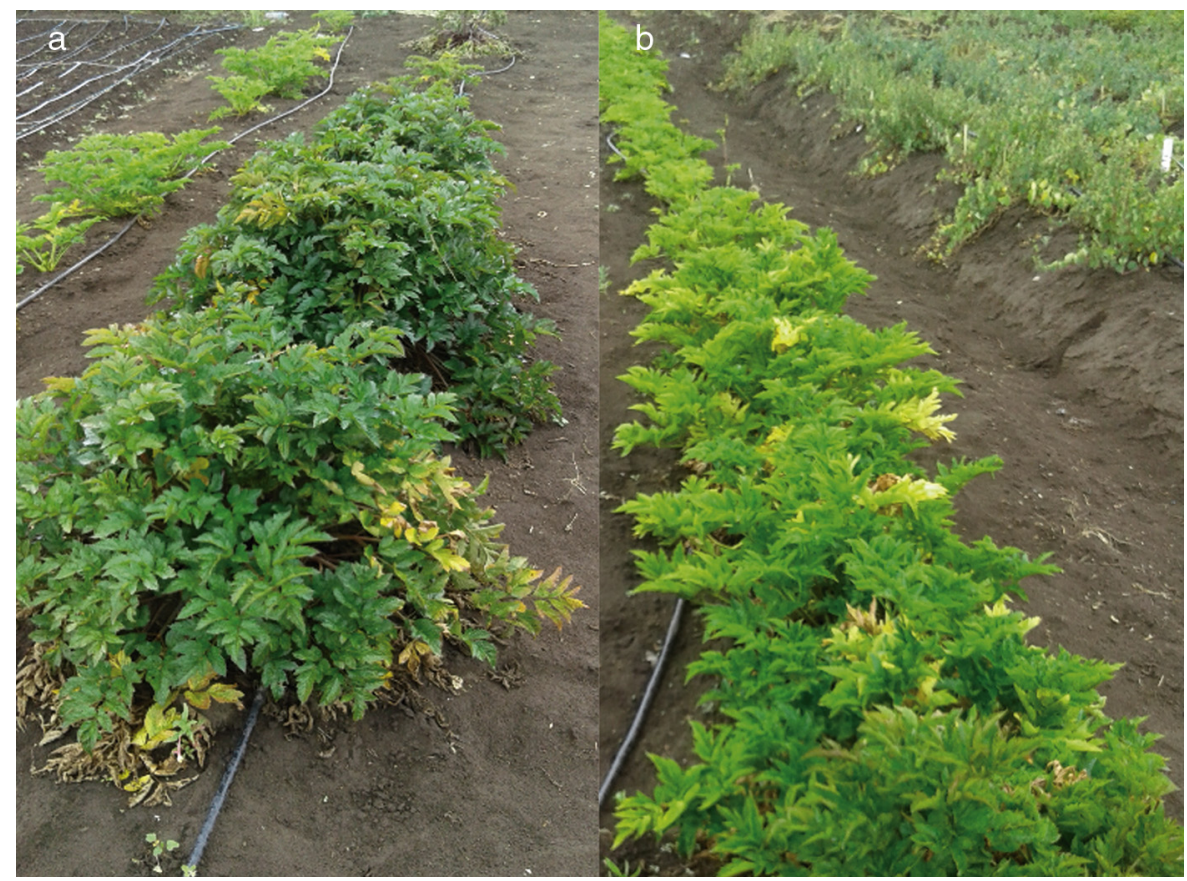

Figura 1. Accesiones de Arracacia xanthorrhiza: de peciolos y nervaduras púrpura (a) y de peciolos y nervaduras verdes (b). 


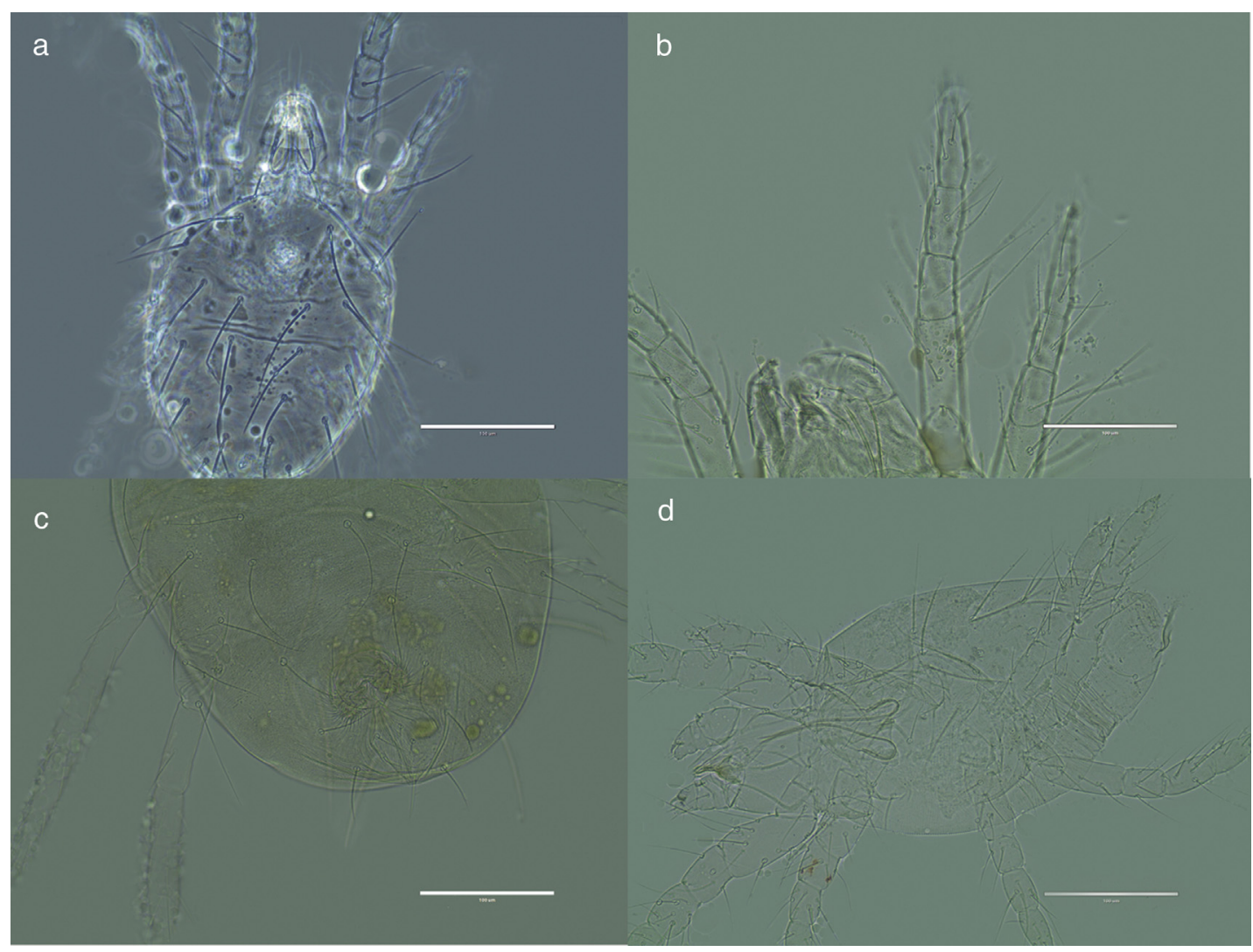

Figura 2. Aspecto general de la hembra de Eotetranychu lewisi (a); sedas dúplex sobre el tarso I (b); placa anal con 2 pares de sedas anales y 2 pares de sedas paraanales (c); edeago curvado ventralmente y reducido abruptamente a una punta delgada (d).

Posiblemente, la presencia de esta especie de ácaro en este cultivo podría estar relacionada con variaciones en la composición fitoquímica de cada accesión, aunque hasta la fecha no existen estudios que respalden esta hipótesis.

Este hallazgo constituye el primer reporte de E. lewisi en la región andina del Ecuador y además se registra por primera vez A. xanthorrhiza (zanahoria blanca) y T. tuberosum (mashua) como hospederas de esta especie de ácaro. Considerando que tanto la zanahoria blanca como la mashua son apreciadas por su valor como componente importante de la dieta en zonas rurales de la sierra ecuatoriana, se recomienda que se hagan estudios sobre el impacto económico en estos y otros cultivos andinos de importancia económica. Por otra parte, considerando el hecho de que E. lewisi es una de las principales plagas en $P$. persica L. en la región centro-norte de México (Pérez-Santiago et al., 2007), y que además ha mostrado una tendencia a incrementar sus poblaciones en plantaciones de fresa en California, EUA (Howell y Daugovish, 2013), el reciente hallazgo de esta especie de ácaro en la provincia de Tungurahua, donde también se producen ambos cultivos, podría representar un riesgo potencial para los productores. Por ello se recomienda hacer muestreos periódicos en las zonas productoras en la sierra ecuatoriana para establecer un inventario de plantas hospederas y su distribución en la región.

Agradecemos a la Dirección de Investigación y Desarrollo de la Universidad Técnica de Ambato por el financiamiento parcial de este proyecto.

\section{Referencias}

Bolland, H. R., Gutiérrez, J. y Flechtmann, C. H. W. (1998). World catalogue of the spider mite family (Acari: Tetranychidae). Leiden, The Netherlands: Koninklijke Brill NV.

Howell, A. D. y Daugovish, O. (2013). Biological control of Eotetranychus lewisi and Tetranychus urticae (Acari: Tetranychidae) on strawberry by four phytoseiids (Acari: Phytoseiidae). Journal of Economic Entomology, 106, $80-85$.

Jeppson, L. R., Keifer, H. H. y Baker, E. W. (1975). Mites for injurious to economic plants. Berkeley, California: University of California Press.

Migeon, A., Nouguier, E. y Dorkeld, F. (2011). Spider mites web: a comprehensive database for the Tetranychidae. Trends in Acarology., 557-560. Disponible en http://www.montpellier.inra.fr/CBGP/spmweb

Pérez-Santiago, G., Otero-Colina, G., González, V., Ramírez, M., González, H. y López, A. (2007). The population level of Eotetranychus lewisi and the concentration of carbohydrates in peach trees. Experimental and Applied Acarology, 43, 255-263.

Vacante, V. (2016). The handbook of mites of economic plants: identification, bio-ecology and control. Wallingford, Reino Unido: CABI. 